\title{
La preservación del patrimonio educativo en el Colegio Nacional de Buenos Aires
}

\author{
María Gabriela Mayoni, Amalia De Grazia, Eugenia Guidobono, Ana Wortley
}

\begin{abstract}
Resumen: El caso del Colegio Nacional de Buenos Aires plantea una dinámica particular en cuanto a su patrimonio cultural, al no tratarse de una institución concebida como museo. El hecho de que las colecciones todavía se utilizan para la enseñanza, caracteriza y condiciona el tipo de tratamiento o cuidado que se les da a las mismas. Los elementos adquiridos y utilizados a través del tiempo en la Institución, definen la historia de la enseñanza pública y de las ciencias en nuestro país. Éstos, contenidos y divididos en cada uno de sus gabinetes deben ser tratados como parte de una colección en sí mismos y por ende, preservados como tales. El Programa de Preservación y Revalorización de Bienes Culturales tiene, en este caso, el fin de adaptar y plantear las acciones de conservación con nuevos enfoques, para alcanzar un equilibrio entre el aspecto histórico - museable de las colecciones y su uso como material didáctico actual.
\end{abstract}

Palabras clave: Institutos de enseñanza; Patrimonio Cultural; Conservación; Nuevos enfoques; Gestión de colecciones.

\section{Preservação do património educacional no Colégio Nacioal de Buenos Aires}

Resumo: $O$ caso do Colégio Nacional de Buenos Aires apresenta uma dinâmica especial, quanto à sua herança cultural, uma vez que não se trata de uma instituição concebida como museu. $O$ facto de que as colecções são ainda utilizadas para o ensino, caracteriza e determina o tipo de tratamento ou cuidado que lhes é dado. Os objectos comprados e utilizados pela instituição, ao longo do tempo, definem a história da educação pública e da ciência, no nosso país. Estes, contidos e divididos em cada um de seus gabinetes, devem ser tratados como parte de uma colecção em si mesma e, portanto, preservados como tal.Neste caso, o Programa de Preservação e de Revalorização de Bens Culturais tem por fim adaptar e colocar novas abordagens nas acções de conservação, para alcançar um equilíbrio entre o aspecto histórico - museológico das coleções e a sua utilização como material didáctico actual.

Palavras-chave: Instituições de ensino; Património Cultural; Conservação; Novos enfoques; Gestão de colecções.

\section{Educational heritage preservation in the National College of Buenos Aires}

\begin{abstract}
The particular case of the National School of Buenos Aires sets out a peculiar dynamic in regards to its cultural Heritage, since it is not an institution conceived as a museum. The fact that the collections within the school are still used for teaching, characterizes and determines the type of treatment or care that its given to them.The objects that have been acquired and used through time in the Institution, define the history of sciences and public teaching in our country. These objects, contained and divided in their own departments have to be treated as part of a collection within itself, and preserved as such.The Program for Preservation and Revaluation of Cultural Heritage has, in this particular case, the aim of adapt and set out the conservation actions with a new approach in mind in order to achieve a balance between the historical-museum aspect of the collections and its use as a current didactic material.
\end{abstract}

Key words: Teaching institutions; Cultural heritage; Conservation; New approach; Collection management.

\section{Introducción}

El Colegio Nacional de Buenos Aires, que tiene sus orígenes durante las misiones Jesuíticas en nuestro país, es una de las instituciones educativas más trascendentes y de gran trayectoria. Es el tercer Colegio Nacional en manos del Estado a partir de 1863 y fue incorporado definitivamente a 
la Universidad de Buenos Aires en 1911. Su acervo patrimonial se fue incrementando y diezmando en diversos momentos aunque afortunadamente, muchas colecciones y elementos didácticos de gran relevancia se mantienen actualmente.

En la segunda mitad del siglo XIX, la adquisición de elementos didácticos procedentes de fabricantes de alto nivel y prestigio mundial constituirá un particular objetivo en un período de gran desarrollo en la educación pública.

Como en muchos otros casos, las instituciones educativas son una fuente de identificación particular de nuestra sociedad. Aspectos políticos, económicos y de desarrollo social se encuentran íntimamente relacionados con las metodologías y sistemas de enseñanza que tuvieron lugar en los diferentes establecimientos a lo largo de la historia.

En la actualidad encontramos muchas instituciones educativas de enseñanza media, públicas y privadas de amplia trayectoria, que poseen un acervo patrimonial muy importante; éste se constituye no solamente por bienes de carácter edilicio y mueble, sino también por todos los objetos que convivieron y fueron testigos del desarrollo educativo de varias generaciones. Estas instituciones no poseen una misión concreta de preservación y salvaguarda de bienes culturales como un museo, pero sí las mismas necesidades y un acervo de igual trascendencia. Muchos de estos elementos han llegado hasta nuestros días y su comunidad educativa convive y hace uso de ellos diariamente.

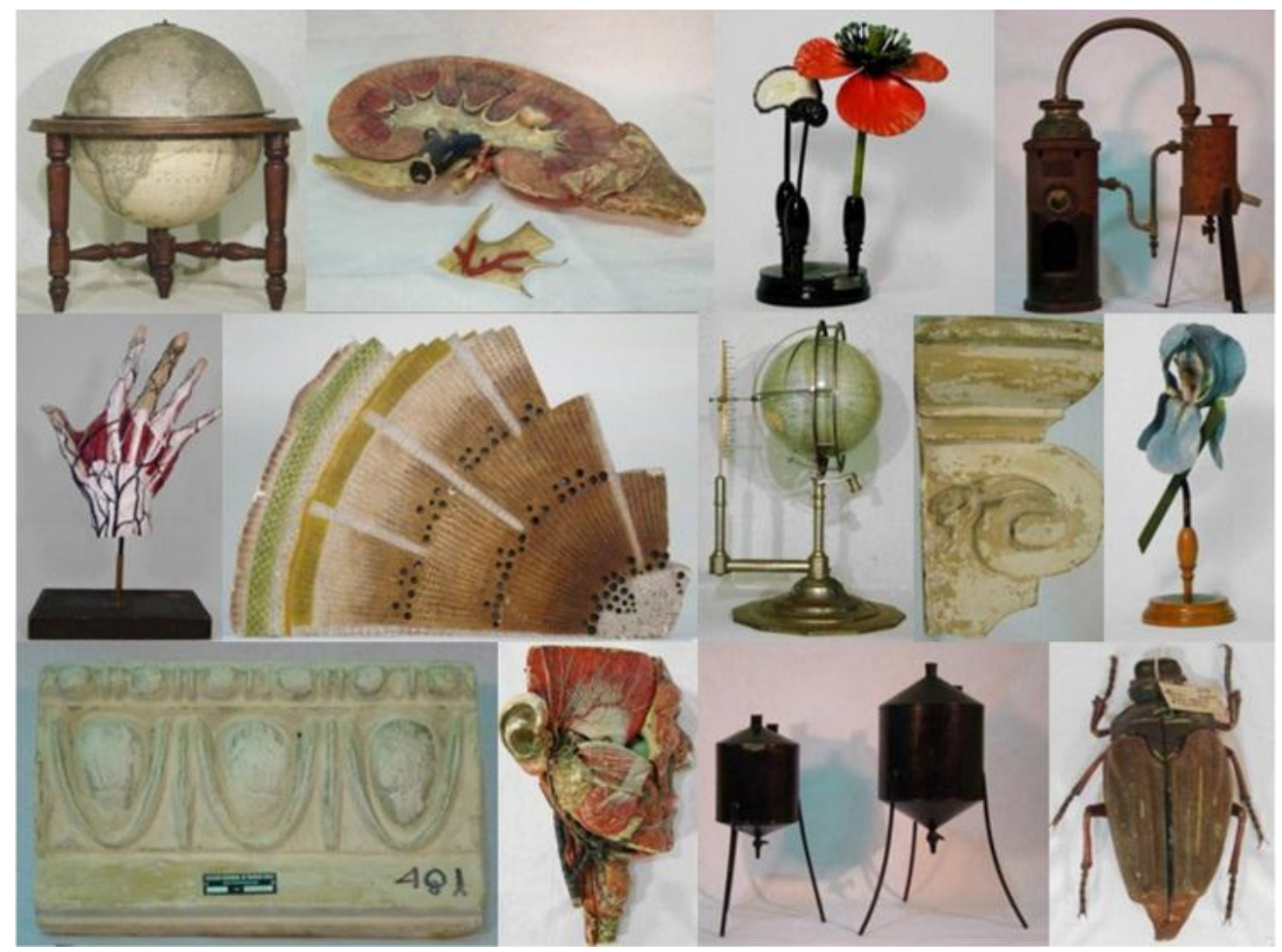


La Revalorización de Bienes Culturales en estos espacios, necesita entonces cumplir en primer lugar con la recuperación y el rescate de su patrimonio como bien cultural.

A partir del año 2007, a través de la incorporación de un equipo de trabajo especializado, tuvieron lugar en el Colegio Nacional de Buenos Aires, proyectos de Revalorización de Bienes Culturales enfocados en la recuperación del material didáctico histórico de la Institución. El Plan Integral de Preservación diseñado tiene como misión los trabajos de documentación, conservación preventiva y restauración de los bienes culturales, así como la difusión de los criterios utilizados y de toda aquella información necesaria para el entendimiento de los procesos realizados.

Uno de los objetivos fundamentales fue la comunicación y la concienciación de su valor mediante la incorporación de conceptos de conservación del patrimonio en el manejo cotidiano de las colecciones.

Hasta el momento los proyectos realizados en el marco del Plan Integral de Preservación y Revalorización de Bienes Culturales del CNBA ${ }^{1}$ son:

Conservación y Restauración de la Colección de Modelos Anatómicos Botánicos Robert Brendel. Departamento de Biología.

Conservación preventiva de la Colección de Modelos Anatómicos de Zoología y Anatomía Humana del Dr. Auzoux y É. Deyrolle. Departamento de Biología.

Conservación preventiva de la Colección de Globos Terráqueos y Restauración del Globo terráqueo en relieve. Departamento de Geografía.

Conservación preventiva de la Colección de Mapas. Departamento de Geografía.

Conservación y Restauración de la Colección de Modelos Anatómicos Botánicos É. Deyrolle y Restauración de una selección de la Colección de Modelos Anatómicos de Zoología y Anatomía Humana del Dr. Auzoux. Departamento de Biología.

\section{Las colecciones didácticas en el Colegio Nacional de Buenos Aires}

En el Colegio Nacional de Buenos Aires se destacan importantes colecciones dentro de sus gabinetes, incorporadas entre finales del siglo XIX y principios del siglo XX. La falta de catálogos originales y fechas exactas de incorporación constituyeron la primera dificultad en su análisis, ya que las únicas referencias de identificación encontradas fueron algunas pocas inscripciones sobre los objetos, referidas en general a fabricantes, importadores o fechas de fabricación, en el mejor de los casos.

Diversas fuentes y documentación histórica que se fueron encontrando y bibliografía a la que se pudo luego tener acceso, han ayudado en la datación de las adquisiciones, llegando a la conclusión de que no se trató de una sola incorporación importante, sino que se mantenía una relación con los distribuidores y era regular el pedido de material extranjero, por lo menos hasta mediados de la década de 1930.

Aproximadamente a partir de esta fecha disminuye el incremento de las colecciones y comienza un proceso de deterioro sistemático de las piezas del siglo XIX, debido en la mayoría de los casos a su continua utilización durante todo el siglo XX, la falta de mantenimiento, el desuso por resultar ob- 
soletos y la poca renovación del material. La Institución además ha sufrido a lo largo del tiempo ciertos períodos de gran pérdida de material. ${ }^{2}$

A continuación se detallan las colecciones de los diferentes Gabinetes:

El Departamento de Plástica posee una importante colección de calcos en yeso de escultura clásica griega y romana, material didáctico con el que hoy los alumnos estudian.

En el Departamento de Química se destaca una colección muy grande de instrumentos y dispositivos de laboratorio del tipo demostrativo y verificativo así como las colecciones de instrumental de laboratorio de vidrio y cerámica.

El Gabinete de Física es el más avanzado dentro de la Institución con respecto a la documentación e investigación de sus colecciones. En el año 2005 se inauguró el Museo Didáctico de Física, creado por docentes con ayuda de alumnos y ex alumnos, cuyas principales actividades son la investigación sobre el funcionamiento de los aparatos.

El Departamento de Geografía posee en su Gabinete varias colecciones destacables: mapas murales, yesos con relieves geográficos, globos terráqueos con información de astronomía y astrología, globos pizarra, elementos etnográficos y una gran colección de minerales, rocas y fósiles.

En el Departamento de Biología, subdividido actualmente en el Gabinete de Botánica y el Gabinete de Zoología y Anatomía Humana, se puede encontrar un acervo comparable a un museo de ciencias naturales. Aún se conservan colecciones de animales embalsamados, preparados histológicos conservados, esqueletos de animales, muestrarios de insectos y mariposas, maderas de diversos lugares, semillas, herbarios, modelos anatómicos de papel maché y de yeso policromado, aparatos de fisiología, diapositivas de vidrio y láminas murales explicativas, entre otros.

Dentro de la Institución diversas ideas y metodologías de trabajo fueron modificando los usos de las colecciones didácticas de cada disciplina. En la actualidad muchas de las piezas son utilizadas por los docentes en las aulas. Con el correr de los años, ya más de cien, los cambios en los sistemas de enseñanza y los diferentes paradigmas en el caso de las ciencias, fueron modificando la función didáctica de los elementos.

\section{Particularidad de este tipo de colecciones}

La particularidad de las colecciones, tal como las que componen el patrimonio de los diferentes gabinetes del CNBA, reside en su carácter como objetos o bienes de uso. A pesar de que la mayoría de estas colecciones pueden ser apreciadas como piezas de relevancia histórica y estética, comparables a otros objetos museables y colecciones de museos internacionales especializados, el caso del CNBA es diferente. Los objetos allí aún mantienen, en la mayoría de los casos, la función original para la cual fueron creados. Muchos objetos mantienen su finalidad didáctica intacta hasta el día de hoy y no se han alejado de la función primordial de la enseñanza. Así, todas las acciones que se lleven a cabo con el objetivo de preservar este tipo de piezas, deben realizarse contemplando esta característica.

Al tratarse de una colección de uso activo, la actitud adoptada por aquellos que la utilizan no siempre va de la mano con la visión del conservador. Las colecciones de este tipo tienen como finalidad ser utilizadas para la enseñanza de una disciplina y de formar parte de una clase, y esto, no siempre significa que serán utilizadas con ciertos cuidados. Es aquí donde reside la principal característica y 
dicotomía de este tipo de objetos, y es allí donde la función del conservador no siempre puede prevalecer, sino que debe a veces renunciar a ciertas exigencias y lograr un equilibrio con aquellas personas más cercanas al objeto. Se pueden realizar sugerencias y recomendaciones, pero resulta claro que no se pueden adoptar con total libertad ciertos criterios que en otros casos resultarían posibles.

La concepción y establecimiento de criterios de conservación y restauración planteados para este tipo de colecciones debe adaptar ciertos aspectos de la conservación restauración tradicionales pero, en su esencia, respetan al máximo la premisa de "¿Para qué se conserva?" Para que dichos materiales puedan ser contemplados y utilizados por las generaciones actuales y futuras. En ese caso esto significa que puedan ser manipulados, desarmados, observados en sus detalles y utilizados de forma activa.

Ejemplo de esto es el caso de la colección de modelos anatómicos botánicos de la firma Robert Brendel, pertenecientes al Departamento de Biología donde, en el marco del Proyecto de Revalorización, se realizó la restauración de las 66 piezas que la conforman, ya que se encontraban muy deterioradas y son aún utilizadas en las clases. En la construcción de los criterios de intervención y al momento de evaluar su lectura visual y morfológica, se tomó en consideración su función didáctica actual.

\section{El criterio utilizado como conservadores restauradores}

Muchas veces, el cuidado de los objetos está relacionado con la actitud colectiva que se tiene hacia ellos, causada por diversos factores como la falta de recursos, falta de espacio, falta de conocimiento, entre otros, que termina siendo repetida y prolongada en el tiempo. Mejorar la condición de una colección puede traducirse en varias cosas: un nuevo inventario, un nuevo sistema de numeración para la identificación de los bienes, un orden de ubicación, un nuevo sistema de almacenamiento como contenedores o mobiliarios especiales que ayudan a tomar conciencia de la fragilidad de las piezas, la limitación en la manipulación, la visualización completa del objeto. Éstas van a requerir para su funcionamiento cambios en el uso acostumbrado, en los criterios de búsqueda y el acceso a los objetos, lo cual seguramente redundará en actitudes más atentas, aunque sea en principio sólo por necesidad de acceder a la colección. Cada acción va a exigir conocimiento, mayor cuidado en la manipulación, mayor atención en el estado de conservación y en una nueva organización.

La opción más clara para el conservador es la de no utilizar más el objeto, pero ¿puede sugerirlo? Éste y otros cuestionamientos quedan como interrogantes de este tipo de proyectos y de la posibilidad o no de ofrecer a aquellos que trabajan este material otro tipo de soluciones que puedan ser mantenidas en el tiempo.

En el caso del Gabinete de Geografía, la colección de mapas cuenta con unos 816 ejemplares, de los cuales se utilizan alrededor de unos 240 . La colección que no se encontraba en uso, se hallaba dispuesta en un ambiente destinado como área de depósito, donde los mapas se disponían de manera desordenada en soportes antiguos de madera. Aquellos mapas de uso cotidiano se encontraban ubicados en otro espacio más pequeño.

El carácter de esta colección es activo, en el sentido en que se va renovando constantemente, ya que periódicamente nuevos mapas se adquieren y algunos otros se dejan de utilizar. Además, se constituye de manera muy heterogénea con mapas actuales, otros de treinta años de antigüedad y 
otros más antiguos, como del siglo XIX, principios del siglo XX, variando en tamaño, manufactura y materiales.

El gabinete contaba ya con un inventario de los mapas de la colección, el cual se tuvo en cuenta para la confección de uno nuevo, pero se complementó con una documentación técnica que reflejara las necesidades de la colección, especialmente en cuanto a su estado de conservación.

Resultó claro, a la hora de encarar el planeamiento de las acciones de conservación preventiva, que la colección necesitaba un profundo proceso de reorganización, ya que se desconocía exactamente el número total e incluso la existencia de algunos mapas. El mal estado de conservación, sumado al hecho de que la colección se encontraba en su mayoría desorganizada e inaccesible, conllevó a que sólo se conociera o se tuviera en cuenta un porcentaje pequeño del conjunto.

Todas las acciones de conservación y determinaciones frente a esta colección se realizaron con el consenso del personal del gabinete, los cuales fueron expresando sus necesidades y preferencias a lo largo del proyecto.

El primer paso fue inspeccionar la colección utilizando como base el inventario y la división en grupos geográficos ya existente. A medida que se fue realizando esta operación, se decidió subdividir los grupos profundizando la clasificación dentro de la colección. Resultó además, que los números de inventario utilizados hasta ese momento no reflejaban la cantidad de mapas reales ni el grupo al que pertenecía cada uno. Así, se determinó disponer un nuevo número de inventario alfanumérico, que reflejase no sólo el grupo al cual pertenece sino también la cantidad de mapas totales dentro de cada grupo.

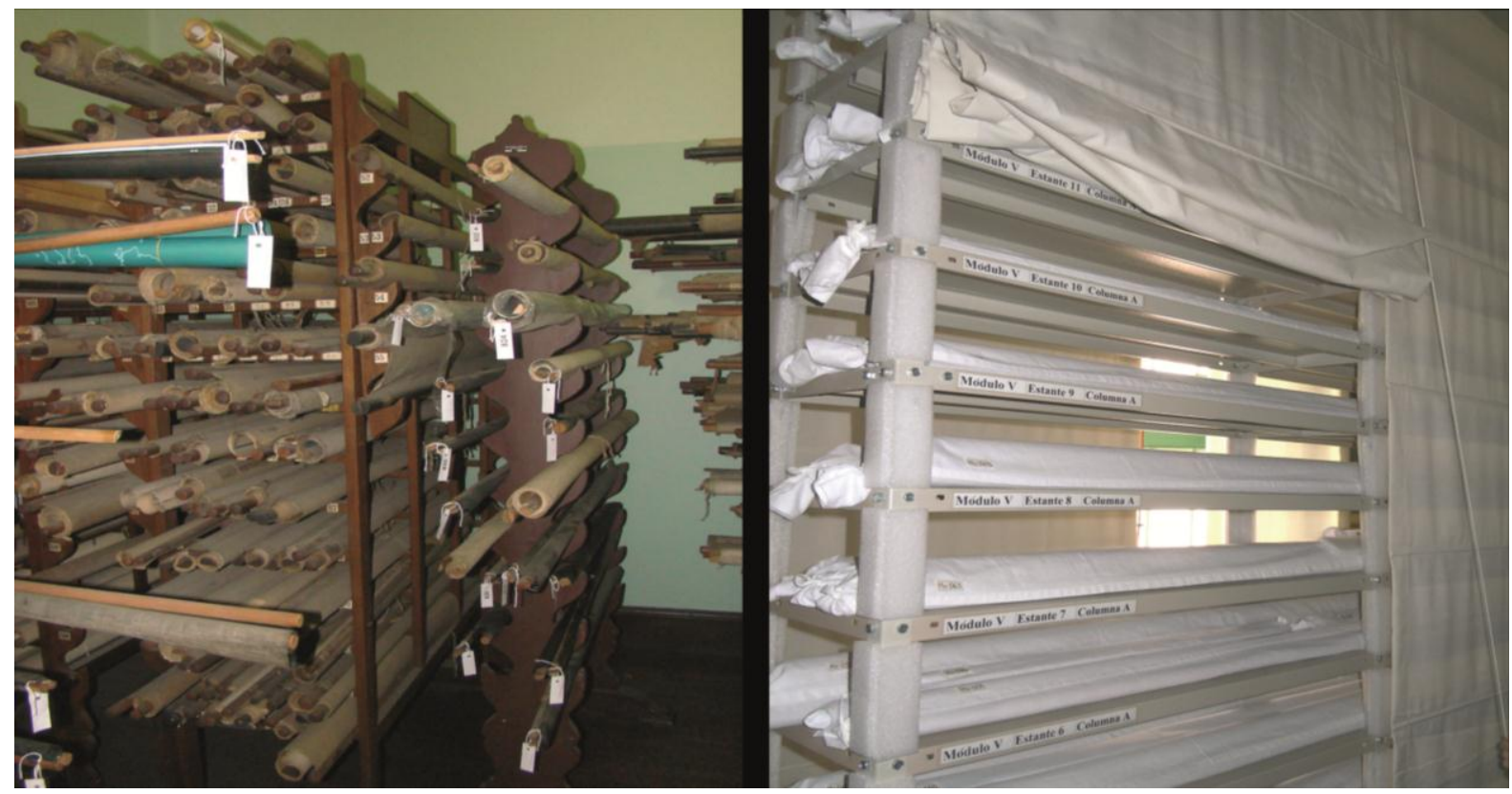

Figura 2. Antes y después de la reorganización de la mapoteca del Gabinete de Geografía. Colegio Nacional de Buenos Aires. 
Los tratamientos de conservación aplicados incluyeron: una ficha técnica para cada uno de los mapas, la cual fue luego digitalizada; una limpieza superficial cuya profundidad dependió del estado de conservación del mapa; el rotulado con el nuevo número de inventario y la confección de fundas para cada uno de ellos.

Otro de los mayores cambios llevados a cabo en esta colección, fue la alteración y mejora de su espacio de almacenamiento en el mismo emplazamiento donde se encontraban anteriormente, logrando una optimización del mismo y teniendo en cuenta la cantidad total de mapas que se albergarían allí. El replanteamiento de los sistemas de almacenamiento, tuvo como premisa la utilización de materiales inertes adecuados para la conservación de los objetos y el establecimiento de condiciones que propicien un mejor manejo y acceso de la colección.

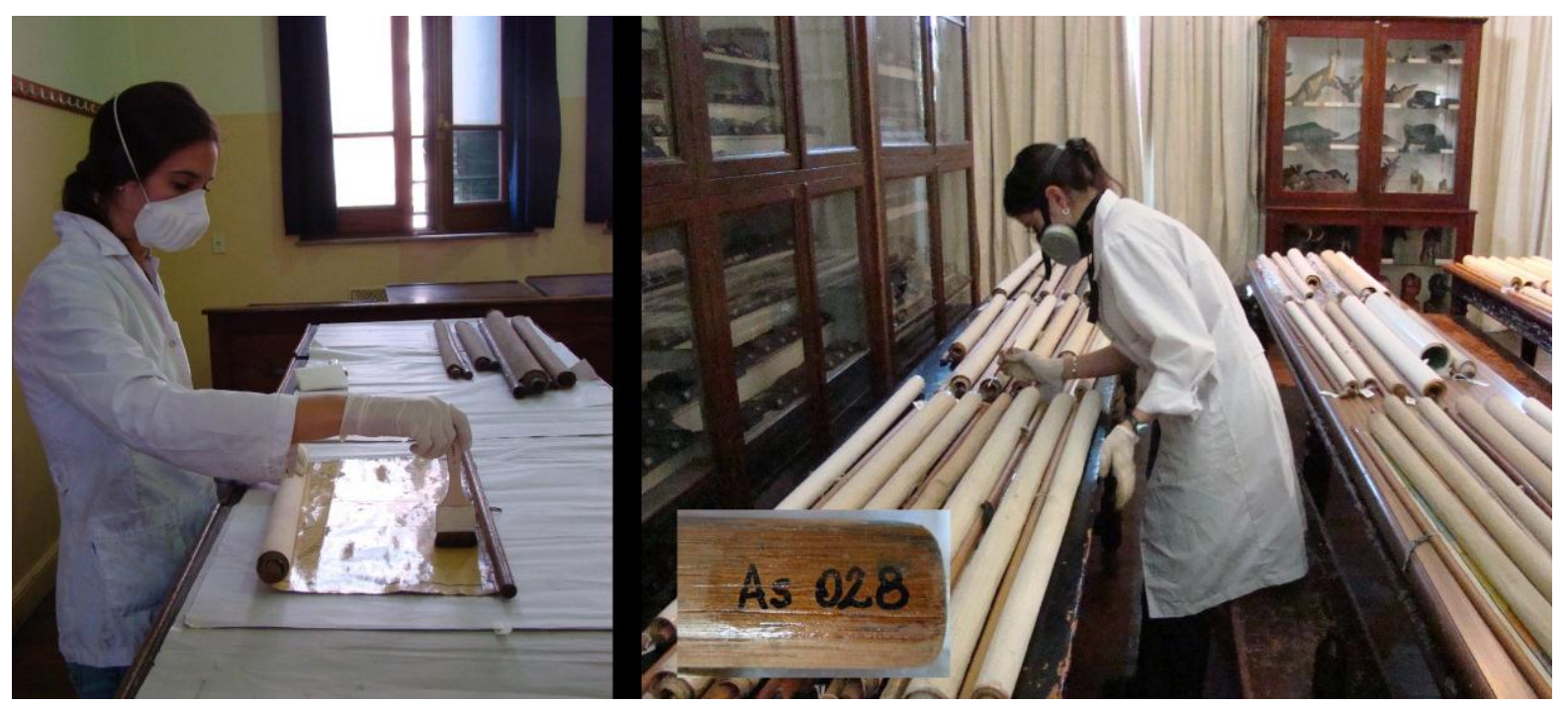

Figura 3. Tareas de conservación, limpieza y rotulado de mapas del Gabinete de Geografía.

El tipo de reorganización llevada a cabo en el Gabinete de Geografía del CNBA resultó un desafío ya que planteó una nueva organización, no sólo en relación con su estado de conservación y su espacio físico, sino también con el comportamiento de los integrantes del gabinete, a los cuales se les modificó el acceso a los objetos y su mecanismo de búsqueda, ahora integrado por una planilla donde se volcó toda la información pertinente.

En el caso de la colección de modelos anatómicos del Gabinete de Zoología también se necesitó lograr una optimización y una organización de su espacio de almacenamiento y un inventario completo con la documentación de cada pieza. Este proceso contribuyó a encontrar elementos que hasta el momento se hallaban perdidos o sin identificación que eran parte de otros objetos más grandes. La reorganización de los sistemas de almacenamiento permitió a los docentes el acceso a piezas clásticas ${ }^{3}$ completas que anteriormente no se conocían o se utilizaban de manera parcial.

El impacto visual en la optimización de los espacios y la utilización de sistemas especiales para albergar y conservar las colecciones, enfatiza la importancia y la conciencia sobre su cuidado. Pasar de un espacio con las piezas desordenadas sin un lugar en particular, a un lugar optimizado para 
un grupo seleccionado, compartimentado, con un lugar específico para cada pieza, con carteles indicadores, fotografías para identificación de los objetos, entre otros, es un contraste que llama la atención y estimula el cambio de hábitos por parte de los docentes, que en esta experiencia comenzaron a manipular las piezas según las reglas que la nueva disposición exigía.

Si bien algunos criterios de conservación dentro de los proyectos se adaptan a las necesidades de la institución y a la función didáctica de las piezas, esto no sucede con otros aspectos fundamentales dentro del proceso de intervención o restauración, tales como el respeto por la originalidad del objeto y los estudios científicos.

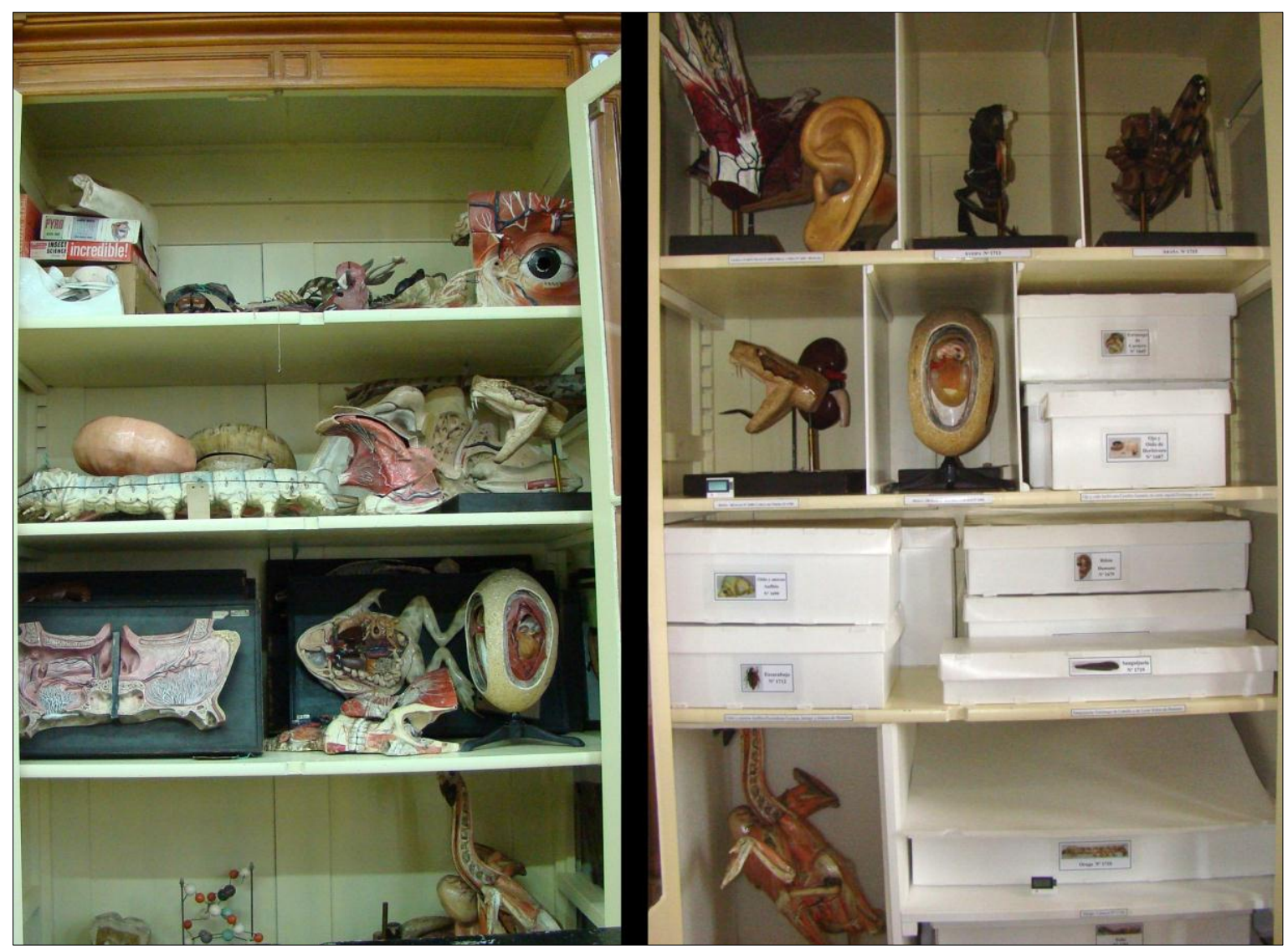

Figura 4. Reorganización del espacio y nuevos sistemas de almacenamiento en el Gabinete de Zoología y Anatomía Humana del Departamento de Biología para la colección de modelos anatómicos.

No se han hecho reconstrucciones sobre los objetos en faltantes que no significaran un impedimento en la correcta lectura y comprensión didáctica de los mismos, ya que se sostiene el criterio de mínima intervención. Sí se hicieron reintegraciones en faltantes a nivel estructural y de elementos relevantes según la necesidad didáctica dentro de las clases; ambos casos, se hicieron con el apoyo de documentación y bajo las indicaciones de los docentes del gabinete respecto a las características morfológicas.

En cuanto a estudios científicos, el ejemplo más destacable dentro de los últimos proyectos realizados es el del Gabinete de Zoología y los modelos anatómicos del Dr. Auzoux. Como en todos los demás casos, se llevó a cabo la observación de muestras al microscopio utilizando los instrumentos 
existentes en el gabinete del colegio e invitando a los ayudantes a participar y observar. Además, se realizó el análisis de muestras con la técnica de Microscopía Electrónica de Barrido ${ }^{4}$ para conocer la composición de pigmentos y ligantes originales. Finalmente se hicieron exámenes de Rayos $\mathrm{X}$ y de Tomografía Computada ${ }^{5}$ de tres modelos para poder conocer con exactitud la estructura interna de construcción de los mismos. Todas estas técnicas, presentadas a la comunidad educativa, generaron un acercamiento diferente hacia los objetos, conociendo otros aspectos y la importancia de estos estudios como parte integral de los proyectos de conservación.

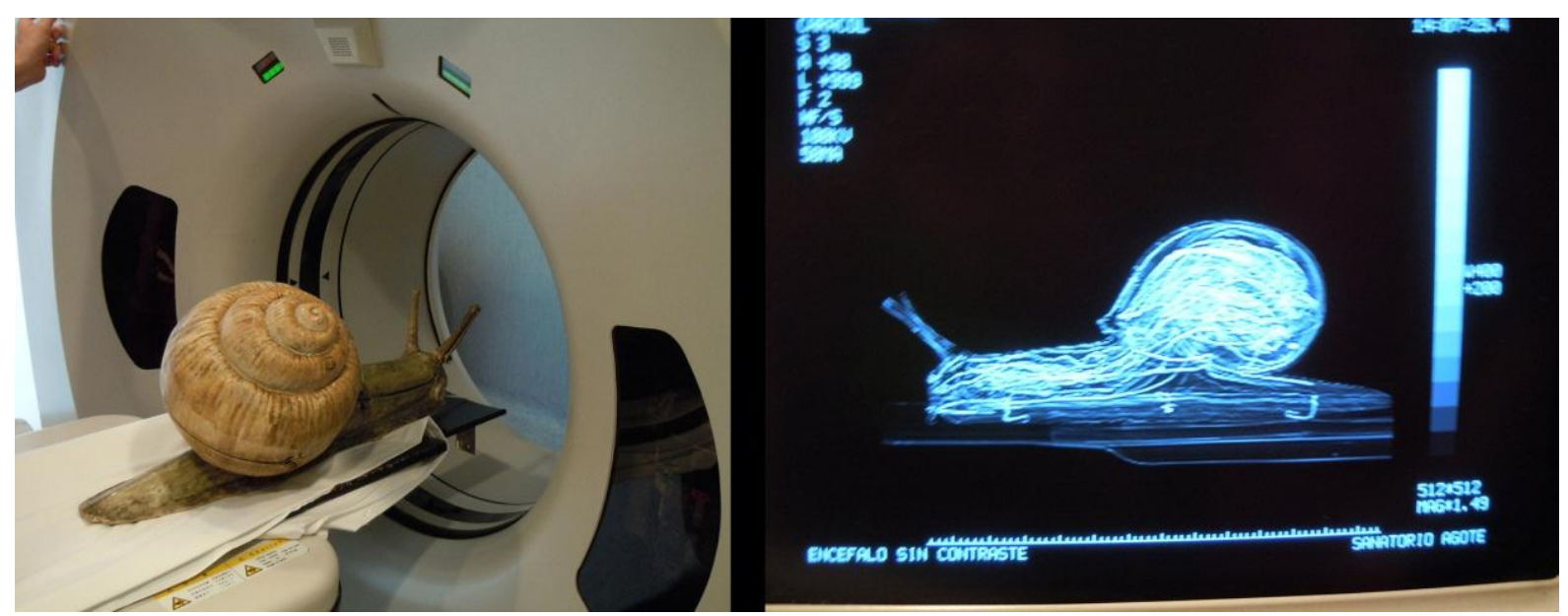

Figura 5. Modelo anatómico de Caracol ingresando al tomógrafo e imagen digital de estructura interna.

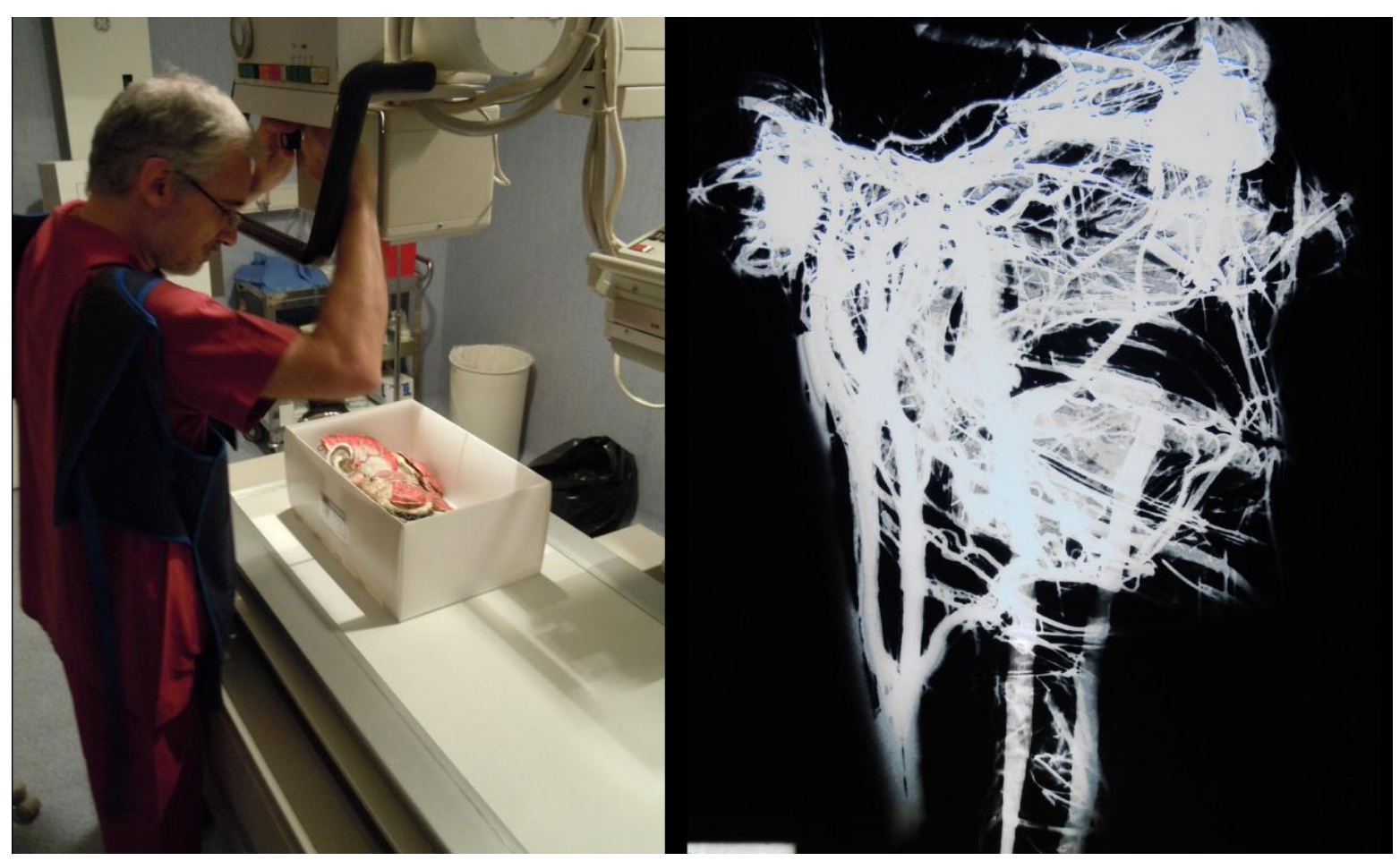

Figura 6. Modelo anatómico de organografía de cabeza humana examinada con un aparato médico de RX y radiografía. 


\section{Concientización y difusión de los criterios}

Como hemos mencionado anteriormente, los objetos que forman parte de las colecciones de los distintos gabinetes son manipulados a diario por docentes de la institución, y aunque en este momento estén siendo intervenidos por profesionales de la conservación, son los mismos docentes quienes deberán manipularlos para el desarrollo de sus clases.

Por este motivo se consideró una prioridad dentro de los proyectos de conservación, la difusión y concienciación a la comunidad educativa, acerca de la importancia del cuidado de los objetos, tanto en su manipulación como en su almacenaje. A estos fines se desarrollaron charlas informativas que acompañadas del contacto diario y el trabajo en equipo con el personal de conservación ayudaron a implementar ciertas pautas de conservación preventiva que se aplican durante y después de las clases en el manejo de estas colecciones.

La importancia de la concientización de la comunidad docente del colegio no sólo es primordial en cuanto lo que se refiere a las acciones preventivas ante el deterioro de los objetos que éstos puedan realizar, sino que se extiende más allá, llegando a los alumnos del colegio, quienes a través del ejemplo y las enseñanzas de los profesores han aprendido a reconocer estas piezas no sólo como material didáctico sino como parte de su patrimonio cultural.

Todos estos conceptos en cuanto al manejo y manipulación de los objetos parecen muy comunes para quienes trabajamos en el campo de la conservación y de los museos, pero son nuevos para muchas personas que tratan con objetos patrimoniales diariamente en otro tipo de instituciones.

La comunidad educativa del CNBA ha incentivado y colaborado con las tareas de conservación de las colecciones desde el comienzo de estas actividades. Han comprendido, a lo largo del desarrollo de los proyectos de recuperación de las colecciones, la importancia de los objetos que forman parte del patrimonio del colegio y han aceptado de manera positiva los cambios que se dieron en los gabinetes a fin de preservar de manera adecuada las colecciones, incluso cuando estos cambios interrumpían o modificaban algunas de sus actividades habituales. La positiva repercusión en la comunidad docente evidencia que la misma no carece de interés o voluntad sino que en el pasado no se les han brindado los conocimientos y las herramientas necesarias para el cuidado adecuado de su patrimonio.

Además, el hecho de que los proyectos estén financiados por la Asociación Cooperadora de padres de alumnos de la Institución, no es un tema menor y es reflejo del interés de toda la comunidad por mantener el legado cultural de estos bienes para las generaciones futuras de estudiantes.

Parte de esta experiencia fueron las actividades realizadas en el marco del Proyecto de Revalorización de Bienes Culturales para el Departamento de Biología, donde fueron convocados alumnos y docentes del Gabinete de Botánica para la primera etapa del proyecto, la investigación y la documentación, participando en la clasificación y catalogación de la colección. De esta manera se permitió el contacto directo de los alumnos con las piezas patrimoniales, propiciando el conocimiento de la relevancia histórica y cultural de las colecciones.

Aprovechando la organización de estos encuentros se estableció otorgar un espacio dedicado a charlas sobre patrimonio y temas relacionados a la preservación, fomentando así en los jóvenes una mayor conciencia hacia sus bienes culturales. 


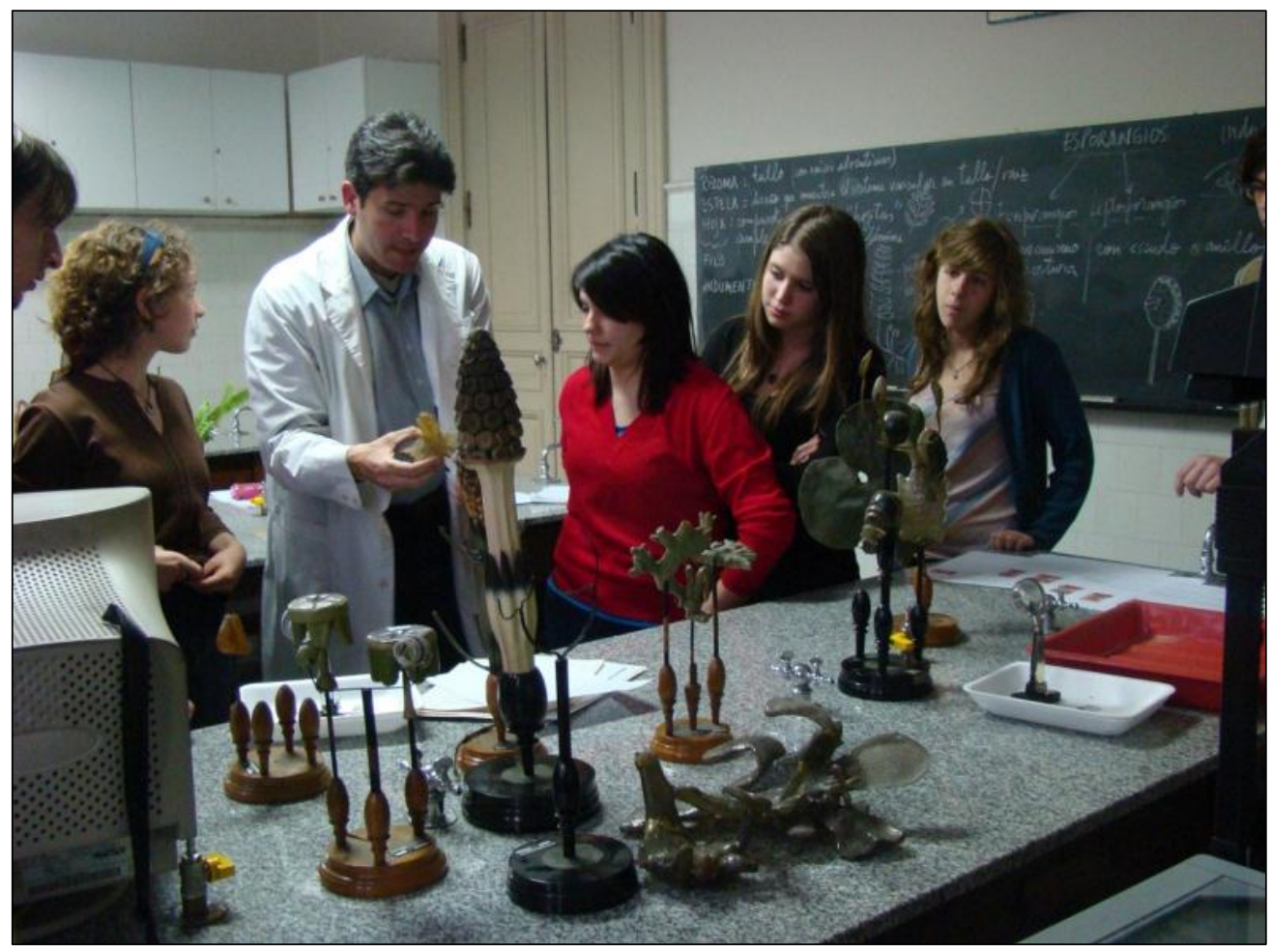

Figura 7. Taller de Taxonomía. Gabinete de Botánica.

Otras actividades para la difusión e integración de la comunidad educativa al proyecto, fueron una serie de charlas de oficios, de técnicas y de materiales, referidas a los procesos tradicionales de construcción de los modelos anatómicos. Estas charlas se realizaron como base e introducción para la propuesta del Taller de Réplicas que se generó en conjunto con los docentes del Departamento de Plástica, realizado con la intención de introducir al alumno en el conocimiento y valorización de las prácticas artísticas involucradas en la confección de estos objetos durante el siglo XIX. La colaboración de docentes y de ayudantes que se comprometieron con la actividad sirvió para hacer frente al manejo pedagógico de los grupos.

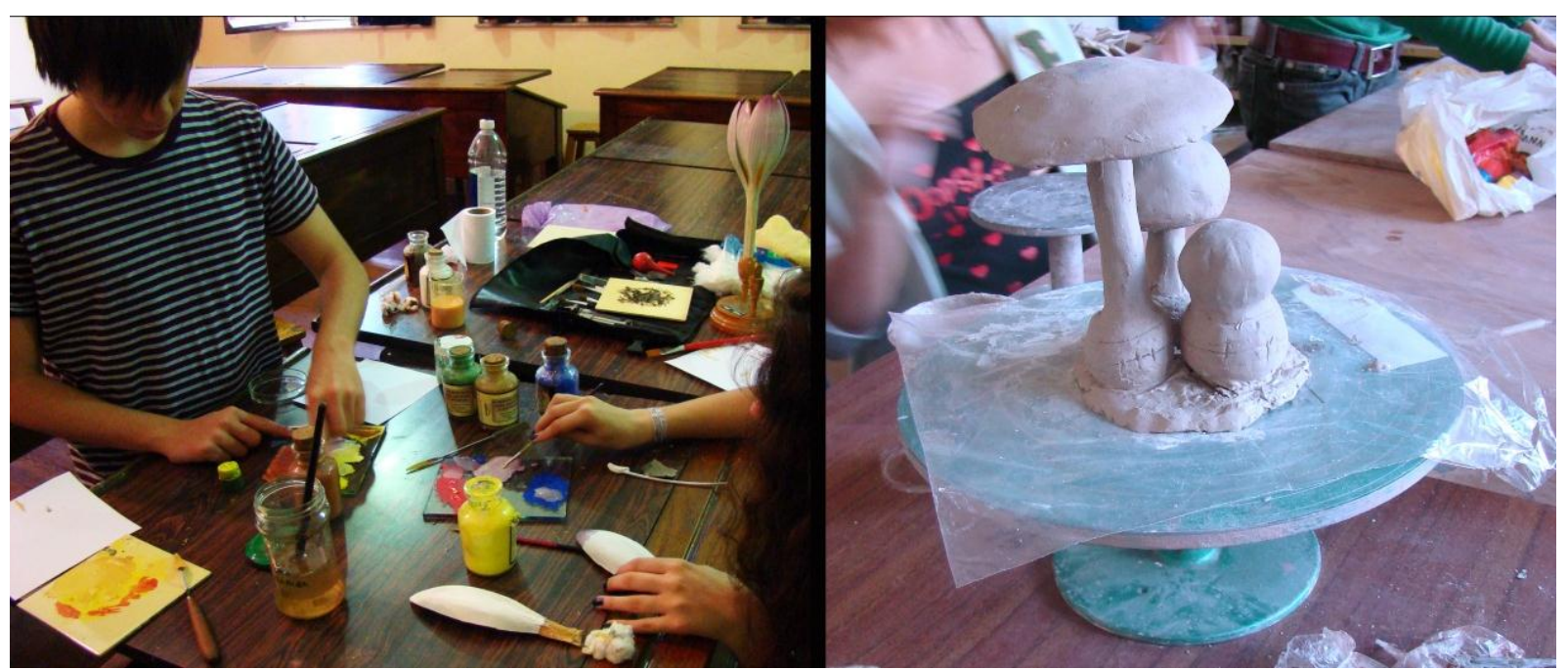

Figura 8. Taller de Réplicas realizado con la colaboración del taller de cerámica y docentes del Departamento de Plástica. $2008-2009$. 


\section{Gestión Cultural}

A fin de completar el recorrido en el proceso de revalorización, la difusión hacia el exterior de la Institución es actualmente un aspecto importante en la planificación de las actividades. Tanto mantener actualizada la información de los trabajos realizados en la página web oficial como la planificación de exposiciones permiten que toda la sociedad en general conozca este tipo de patrimonio y su valorización como elemento de identidad cultural. Esto logró ponerse de manifiesto con la exhibición de la colección de modelos anatómicos botánicos, dónde se realizó un trabajo interdisciplinario y se trabajó en un guión museológico que articulase las diferentes facetas del proyecto. La difusión y la investigación de la colección fueron el hilo conductor principal, enmarcando las diferentes actividades de conservación y restauración y enfatizando la particularidad de esas acciones en una institución que no es museo, pero que tiene un acervo de gran valor e importancia social y cultural.

Es importante mencionar, además, que el estudio del patrimonio histórico educativo posee pocos antecedentes, constituyéndose una línea de investigación emergente entre historiadores de la educación y de las ciencias y una nueva rama de especialización técnica para los profesionales de la conservación.

Por eso mismo, en el marco de la difusión, el intercambio de experiencias e información entre especialistas de las diferentes áreas involucradas es, hoy en día, la herramienta fundamental para la incorporación de nuevos conocimientos y la construcción de criterios para su preservación. Actualmente, instituciones educativas y museos de ciencias de diferentes países poseen ya en exhibición este tipo de patrimonio cultural, siendo objeto de acciones de conservación, intervención y difusión a la sociedad. Por otro lado, se puede mencionar la creación de agrupaciones y redes de investigadores e instituciones que se constituyen como verdaderas plataformas para los procesos de revalorización y la divulgación del patrimonio ${ }^{6}$.

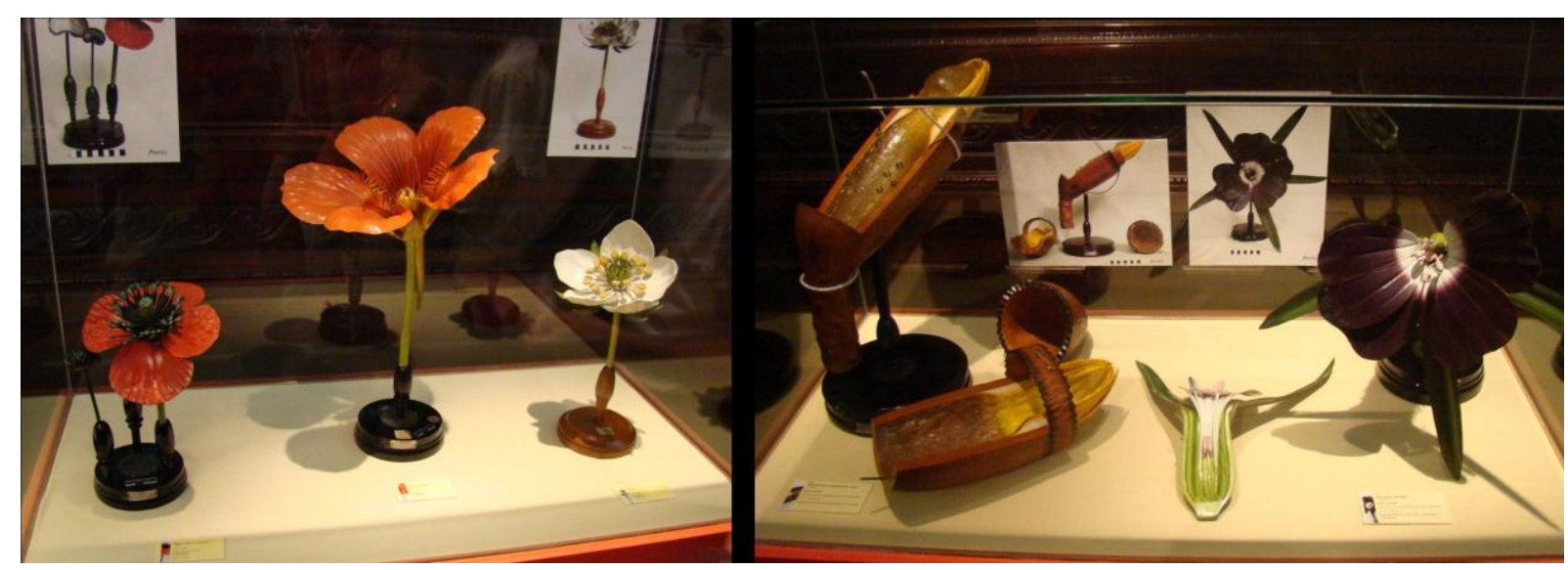

Figura 9. Exhibición "Modelos del Pasado". Preservación de Modelos Anatómicos Botánicos - Colección Robert Brendel. Comisión Nacional de la Manzana de las Luces. Buenos Aires. Noviembre 2009. 


\section{Conclusiones}

En el Colegio Nacional de Buenos Aires, uno de los aspectos más satisfactorios a la hora de realizar proyectos de preservación sobre las colecciones didácticas es la toma de conciencia que se genera en la comunidad educativa, docentes y alumnos, entorno al objeto. Éste, contemplado antes tan sólo como mero ejemplo o soporte de una clase, pasa a estar enmarcado en un proyecto de revalorización, hecho que es visto y tenido en cuenta por aquellos que trabajan diariamente con las colecciones. Se ha observado, una vez finalizados los trabajos dentro de un gabinete, que el docente se aproxima a éste de otra manera, correspondiente con el cuidado implícito en su conservación. Tareas simples como la utilización de guantes a la hora de tocar un objeto o el almacenaje apropiado en sus respectivos contenedores, dan cuenta de que la actitud de los docentes y por ende de los alumnos que lo observan ha sufrido un cambio positivo en cuanto a la aproximación al objeto.

Todas las actividades que se puedan realizar en conjunto son vitales y ayudan al cambio de perspectiva en la concepción de las colecciones, más aún cuando es necesario un cambio de nivel, como en este caso, de bien utilitario a bien cultural. Sentirse partícipes de la protección del patrimonio también propicia la atención y el interés por proteger y cuidar los bienes culturales que forman parte de la historia de la comunidad. Afortunadamente, el apoyo y entusiasmo de los diferentes agentes involucrados propició el desarrollo de nuevos planes de preservación y revalorización de material didáctico, pudiendo mejorar la situación de otras colecciones.

El objetivo final de todos estos procesos es que desde la base la valorización sea la correcta y que todas las acciones realizadas sirvan a largo plazo. Uno de los conceptos que se intentó destacar es que todo lo que se logra en el presente es beneficioso para el patrimonio en el futuro, toda la información que se rescata de las colecciones afianza su seguridad y el sentido de pertenencia, limitando la posibilidad de nuevas pérdidas. Lo importante es no volver atrás en el camino, sino afianzar las bases para el avance de acciones y así poder ir progresando en la puesta en valor del patrimonio cultural.

\section{Agradecimientos}

A la Asociación Cooperadora "Amadeo Jacques" del Colegio Nacional de Buenos Aires, a las autoridades de la Institución, docentes y alumnos de los gabinetes involucrados, a toda la comunidad educativa en general y los colaboradores del proyecto que nos han acompañado en las diferentes etapas. A colegas y otros profesionales que han tenido interés por nuestro trabajo dentro y fuera del país, ayudándonos en su difusión.

\section{Notas}

[1] En el espacio otorgado en la página web oficial para el Grupo Conservación-Restauración, están a disposición los informes realizados de cada proyecto, así como los criterios utilizados y una galería de imágenes con algunas colecciones trabajadas. www.cnba.uba.ar/conservacion

[2] Según varios testimonios, en la década del 1960 se pintó por dentro todo el edificio, llevando a los gabinetes a una gran limpieza de objetos en desuso y material roto o muy deteriorado. En los pasillos se dejaban las cosas que los gabinetes ya no querían y que ya estaban dados de baja en los libros, ofreciéndolas a los interesados. Lo que no era llevado era desechado. Paradójicamente, los momentos de pérdida masiva de material no estuvieron relacionados con catástrofes del tipo incendio o inundación, sino con la determinación de inutilidad del objeto para seguir cumpliendo con su función didáctica. 
[3] Clástico/ca: adj. Se dice de las piezas anatómicas artificiales de un modelo, desmontables para su estudio. Diccionario de la Lengua Española Real Academia Española. Vigésima segunda edición. http://www.rae.es/rae.html [consulta: 24/02/2012]

[4] Realizadas en el Servicio Geológico Minero Argentino (SEGEMAR).

[5] Realizados en el Sanatorio Dr. Luis Agote, Swiss Medical Group.

[6] En España puede destacarse en relación a instituciones educativas de segunda enseñanza, la Red CEIMES (CCHS-CSIC y UNED), la Asociación Nacional para la Defensa del Patrimonio de los Institutos Históricos (ANDPIH), el Museo de Historia de la Educación "Manuel B. Cossio" y la Red de museos de la Universidad Complutense de Madrid (UCM).

\section{BibLIOGRAFÍA}

BUCCHI, M. (1998). "Images of science in the classroom: wallcharts and science education 1850-1920", British Journal of History of Science, 31:161-184.

DUMONT, B., DUPONT, A-L., PAPILLON, M-C, et. al. (2011). "Technical Study and Conservation Treatment of a Horse Model by Dr. Auzoux", Studies in Conservation, 56: 58-74.

GARCÍA, S. V. (2007). "Museos escolares, colecciones y la enseñanza elemental de las ciencias naturales en la Argentina de fines del siglo XIX.", História, Ciências, Saúde-Manguinhos, 14, 1: 173-196.

(2010b). "Museos y materiales de enseñanza en la Argentina, 1890-1940". En El museo en escena. Políticas culturales y museos en América Latina, Castilla A. (comp.). Buenos Aires: Editorial Paidós.

GÓMEZ R. DE CASTRO, F. (1997). "Lecciones de cosas y centros de interés". En Historia ilustrada del libro escolar en España: del Antiguo Régimen a la segunda República, Escolano Benito, A. (Dir.). Madrid: Fundación Germán Sánchez Ruiz Peréz/Pirámide, 449-466.

GROB, B. (2000). The World of Auzoux: Models of Man and Beast in Papier-Mâché. Leiden: Museum Boerhaave.

GROB, B., NIJHOFF ASSER, E., GIACCONE, E. M., (2008). Papieren Anatomie, De wonsderschone papiermachémodellen van dokter Auzoux. Leiden: Museum Boerhaave, Walburg Pers.

GVIRTZ, S. (2000). El color de lo incoloro. Miradas para pensar la enseñanza de las ciencias. Buenos Aires: Editorial Novedades Educativas.

MAYONI, M. G. (2011). Puesta en Valor de Bienes Culturales en el Colegio Nacional de Buenos Aires. Colección Didáctica de Modelos Anatómicos en Papel Maché del S. XIX. Tesis de grado-Licenciatura en Conservación y Restauración de Bienes Culturales. Buenos Aires: Departamento de Artes Visuales "Prilidiano Pueyrredón", Instituto Universitario Nacional del Arte.

MOTEL, J. (2004). L'Anatomie Clastique et Le Musée de L'Écorché D'Anatomie du Neubourg. Neubourg: Musée de L'Écorché D’Anatomie.

MYERS, J. (1997). Los futuros del pasado. Universidad, ciencia y modernización 1870- 1920. Catálogo de exhibición, Buenos Aires: Museo Etnográfico de la Facultad de Filosofía y Letras, Universidad de Buenos Aires.

REILING, H. (2003). "Beter dan de natuur". En Jan Brand \& Alex de Vries (eds), Utrecht: Utrecht Central Museum, 221-235. 
RUIZ BERRIO, J. (ed.) (2010). El patrimonio histórico-educativo: su conservación y estudio: Madrid, Biblioteca Nueva.

SEDDON, T (1994). "Conservation of Anatomical Botanical Plant Models". En Conservation and the Herbarium, R.E Child (ed). Tunbridge Wells: Institute of Paper Conservation, 29-33.

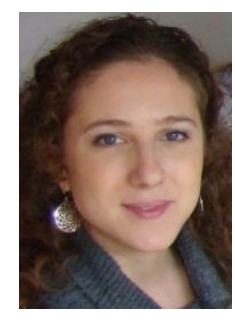

María Gabriela Mayoni

mgmayoni@gmail.com

Licenciada en Conservación-Restauración de Bienes Culturales. Realizó estancias en el Museo Nacional de Artes Decorativas de Madrid como becaria entre el 2010 y 2012. Colaboró con el Museo Veterinario Complutense y el IPCE. Expuso sus trabajos en diferentes encuentros dentro y fuera del país.

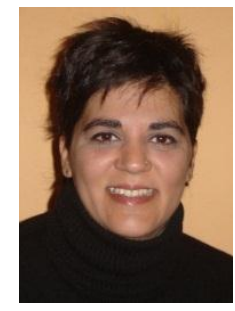

Amalia De Grazia

amaliadegrazia@yahoo.com.ar

Egresada de la Licenciatura en Conservación-Restauración de Bienes. Es conservadora de material gráfico y fotográfico y Coordinadora del Área de Conservación en el Museo del Cine de la Ciudad de Buenos Aires. Realizó estancias en el IPCE y el Museo Reina Sofía. Participó de diferentes encuentros dentro y fuera del país.

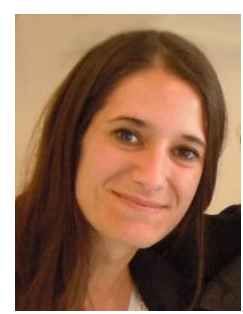

Eugenia Guidobono

eugeguidobono@gmail.com

Licenciada en Conservación-Restauración de Bienes Culturales. Complementó sus estudios con pasantías en el exterior, Fundación para la Conservación del Patrimonio, Caracas, Venezuela. Participó de diferentes cursos y expuso sus investigaciones y trabajos en diferentes encuentros especializados dentro y fuera del país. 


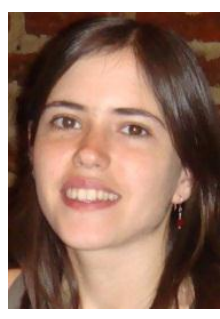

Ana Wortley

anawortley@hotmail.com

Egresada de la Licenciatura en Conservación-Restauración de Bienes Culturales. Realizó pasantías de formación en el exterior en Heritage Malta (Kalkara, Malta) y en el Victoria \& Albert Museum (Londres, Reino Unido). Participó de diferentes congresos, jornadas y encuentros especializados dentro y fuera del país.

Artículo enviado el 08/10/2011

Artículo aceptado el 12/05/2012 